\title{
Bubble trouble: a review of diving physiology and disease
}

\author{
D Z H Levett, ${ }^{1}$ I L Millar ${ }^{2}$
}

${ }^{1}$ Centre for Altitude, Space and Extreme Environment Medicine, UCL, London, UK; ${ }^{2}$ Hyperbaric Service, The Alfred Hospital, Melbourne, Australia

Correspondence to: Dr D Levett, Centre for Altitude, Space and Extreme Environment Medicine, UCL, 1st Floor Charterhouse Building, Archway Campus, Highgate Hill, London N19 5LW, UK; denny.levett@ ucl.ac.uk

Received 14 May 2008 Accepted 18 August 2008

\section{ABSTRACT}

Exposure to the underwater environment for recreational or occupational purposes is increasing. Approximately 7 million divers are active worldwide and 500000 more are training every year. Diving related illnesses are consequently an increasingly common clinical problem with over 1000 cases of decompression illness reported annually in the USA alone. Divers are exposed to a number of physiological risks as a result of the hyperbaric underwater environment including: the toxic effects of hyperbaric gases, the respiratory effects of increased gas density, drowning, hypothermia and bubble related pathophysiology. Understanding the nature of this pathophysiology provides insight into physiological systems under stress and as such may inform translational research relevant to clinical medicine. We will review current diving practice, the physics and physiology of the hyperbaric environment, and the pathophysiology and treatment of diving related diseases. We will discuss current developments in diving research and some potential translational research areas.

Diving exposes humans to immersion and to elevated ambient pressures, which result in a range of physiological effects and potentially pathophysiological sequelae over and above the risk of drowning. Despite these challenges, recreational scuba diving is popular. The world's largest diver training agency, PADI, certifies approximately 500000 new divers annually and it is estimated that around 7 million divers are currently active worldwide. Although modern equipment and training have made diving relatively safe, an average of 100 diving related deaths and 1100 cases of decompression illness are reported annually in the USA alone. ${ }^{1}$ In addition to recreational diving, many dives are undertaken for scientific, seafood harvesting, construction, maintenance, filming, military, forensic and rescue purposes. Currently, the challenging forms of occupational diving associated with offshore oilfield exploration and construction are undergoing a major resurgence.

This review will address:

- the types of diving practised today

- the physical and physiological effects of the underwater environment

- the breathing gases used by divers and their toxic effects

- the aetiology and pathophysiology of diving diseases

- the management principles of diving diseases

- current developments in diving research and areas of translational research.

\section{TYPES OF DIVING}

Diving can be undertaken in three fundamentally different manners: breath-hold diving, "bounce" diving with breathing apparatus, or saturation diving. Breath-hold and bounce diving are regularly used for both recreational and occupational purposes, while the cost and logistic complexity of saturation diving usually limits its use to the high value construction and maintenance work associated with the oil industry.

\section{Breath-hold diving}

This most ancient and basic means of submergence remains a core means of collecting seafood, but it has also recently developed as a competitive sport, often referred to as apnoea diving. There are a number of competitive apnoea diving disciplines depending on the mode of submergence and the type of assistance allowed (for example, fin swimming for ascent or descent, assisted descent with weights, assisted ascent with buoyancy devices). As of January 2008, competitive surface, face immersed breath-hold durations had reached $9 \mathrm{~min}$, horizontal underwater swims with fins, $244 \mathrm{~m}$ distance, and free diving descent with fin propulsion only, $112 \mathrm{~m}$ deep $(1232 \mathrm{kPa})$. A world record $214 \mathrm{~ms}$ (700 feet; $2262 \mathrm{kPa}$ ) "no limits" descent was achieved by Herbert Nitsch in 2007 using a releasable weight to descend rapidly and a non-compressible buoyant and hydrodynamic "hat" to ascend, backed up by high speed winches, in a dive that lasted 4 min $6 \mathrm{s.}^{2}$

\section{Diving with breathing apparatus}

Diving using a helmet supplied with free flowing air has a history of many hundreds of years, but modern recreational and occupational diving has grown from the development of the self contained underwater breathing apparatus (SCUBA), which was pioneered by Cousteau and Gagnan in 1942. The modern single hose demand scuba regulator was subsequently invented by Eldred, and first sold as the Porpoise regulator in 1952. Its elements remain unchanged today: a high pressure air cylinder carried on the back with a two stage demand valve system, which allows the diver to breath air from a mouthpiece with inspiratory and expiratory pressures that vary by only a few centimetres of water pressure above and below ambient. More challenging occupational diving usually uses surface supplied breathing gas and full face masks or helmets equipped with voice communications (surface supplied breathing apparatus, SSBA). The final gas delivery of SSBA systems usually works on the same principle as the demand regulator of the recreational scuba 
diver but some other systems, such as free flow helmets, are in use. Seafood harvesters often use a simplified form of SSBA involving a single floating hose and recreational scuba style half mask and mouth held regulator.

Free swimming diving can also be undertaken using closed circuit rebreather (CCR) apparatus with a carbon dioxide absorbent and oxygen injection to compensate for respiratory consumption. Oxygen only CCRs were developed for bubble-free covert military operations, but the technology has advanced significantly in recent times to involve "trimix" (oxygen, helium and nitrogen) systems controlled by gas analyser cells and computers that enable dives in excess of $100 \mathrm{~m}$ deep for many hours in duration.

\section{Saturation diving}

Saturation diving is used for deeper dives lasting many days and this requires divers to live inside a pressure chamber usually located on a diving support vessel (DSV) or an oil platform. The pressure used approximates that of the depth of water in which the diving work is required and teams of divers are transported from the living chamber to the dive site by a submersible pressurised transport chamber usually referred to as a "diving bell". Two or more teams enable $24 \mathrm{~h}$ underwater productivity over several weeks, at the end of which the diving team is brought to surface pressure with minimal risk of decompression sickness by decompressing the chamber over several days. Since the decompression requirement for saturation divers may be more than a week, pressurised DSVs represent one of the most remote environments on earth.

In addition to the normal diving environments of fresh or salt water, occupational divers are occasionally called to work in fluids as challenging as raw sewage, fermenting cheese whey and radioactive nuclear power station cooling water. Non-immersed pressure exposure also occurs in some tunnelling and caisson work and for patients and clinical staff in hyperbaric oxygen therapy chambers. While much of what follows also applies to these situations, these environments will not be specifically discussed.

\section{PHYSICAL AND PHYSIOLOGICAL EFFECTS OF THE UNDERWATER ENVIRONMENT}

Most physiological challenges of the underwater environment flow from the physics of immersion and the pressure associated with increasing depth.

Immersion causes a central redistribution of blood volume, which is increased if cold water triggers vasoconstriction. This induces antidiuretic hormone $(\mathrm{ADH})$ release and results in a diuresis, rendering the diver relatively hypovolaemic on surfacing. Breathing dry cylinder gas exacerbates any fluid deficit rendering divers at high risk of dehydration which increases their susceptibility to diving related illness.

The rate and quantum of pressure change involved in diving greatly exceed anything that humans normally encounter as a result of altitude change. The ambient pressure at highest point on the earth, the summit of Mount Everest $(8500 \mathrm{~m})$ is approximately one third of the sea level value $(33 \mathrm{kPa})$. In contrast water's density is approximately 1000 times that of sea level air and ambient pressure increases linearly with depth of submergence at a rate of 1 additional atmosphere $(1$ bar or $101 \mathrm{kPa}$ ) for each $10 \mathrm{~m}$ of sea water (msw). As a result, recreational scuba divers diving from sea level to $30 \mathrm{msw}$ experience a fourfold pressure increase from $101 \mathrm{kPa}$ to $404 \mathrm{kPa}$ absolute. The deepest in-water working dives have taken human beings to depths of $534 \mathrm{msw}(5494 \mathrm{kPa})$ while the highest human pressurisation recorded was $701 \mathrm{msw}(7181 \mathrm{kPa})$ by the Comex Hydra 11 team in 1992. ${ }^{3}$ Thus divers are exposed to variations in ambient pressure far in excess of those experienced by high altitude climbers or astronauts.

These pressure changes have profound effects on the behaviour of gases with important implications for divers. The partial pressures exerted by each component of the diver's breathing gas increases proportionally with the total ambient pressure as depth increases (Dalton's law) (fig 1). Exposure to supranormal pressures of gases results in proportionately supranormal quantities of gas being dissolved in body tissues (Henry's law), with profound physiological effects including seizures in the case of oxygen toxicity or impaired cerebral function with nitrogen narcosis (discussed in greater detail below). On returning to the surface, the reduction in ambient pressure results in supersaturation of tissues with gases, which is associated with bubble formation and in some cases pathological consequences.

The increase in pressure with depth also has implications for gas filled tissue spaces as pressure and volume are inversely related (Boyle's law). Unvented gas spaces will be compressed on descent and will expand on ascent if contained within distortable tissues (for example, the bowel, and lungs during breath-hold). In non-distortable tissues such as the middle ear and sinuses, a pressure gradient will develop between any isolated gas and surrounding tissues resulting in barotrauma.

Increased ambient pressure brings with it increased density of breathing gas. This, combined with immersion, brings about reduced pulmonary compliance, increased airways resistance, increased $\mathrm{VQ}$ mismatch, and an increase in the work of breathing. This increase in the work of breathing is one of the factors which limits maximum diving depth. The heliumoxygen mixtures that usually replace air as a breathing mixture for dives over $50 \mathrm{~m}$ are much less dense and have reduced viscosity compared with air. Despite this, the work of breathing at great depths becomes such that inspiratory and expiratory mechanical assistance systems have been developed to enable underwater work. ${ }^{4}$ Hydrogen has also been explored as the least dense diluent for oxygen but adverse neurological effects offset any potential gain over heliox or trimix (helium, oxygen, nitrogen). ${ }^{5}$ Regardless of breathing gas, poor breathing apparatus design, high work rates or failure of absorbent in rebreathing circuits can lead to $\mathrm{CO}_{2}$ retention, especially during deeper

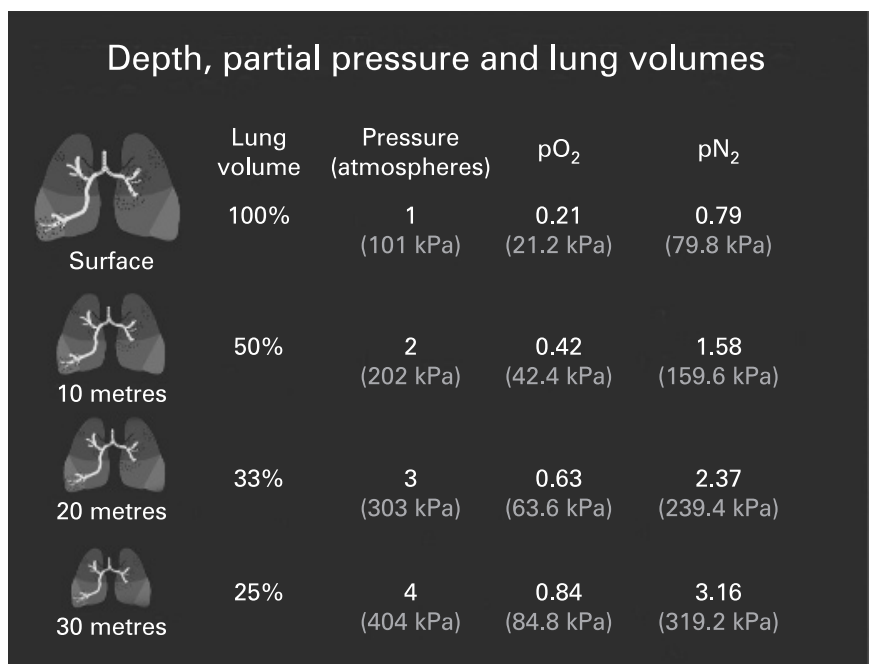

Figure 1 Nominal volume, pressure and partial pressure changes with depth. 
dives, and this has caused a significant number of underwater deaths and "near misses".

A further challenge is that of temperature regulation. The high thermal conductivity and capacity of water and dense breathing gas and the cold water render the diver at risk of hypothermia. This is a particular threat in deep technical dives in non-tropical waters (such as the UK), which necessitate long decompression periods with little activity. Appropriate exposure suits are essential and for all divers to minimise heat loss (dry suits or wet suits). In saturation diving warmed suit systems and warmed breathing gas supplies are often employed to maintain core body temperature.

\section{DIVING GASES: CHARACTERISTICS AND TOXIC EFFECTS}

Although a gas supply is essential for survival in the underwater environment, breathing gas under conditions of increased partial pressures is not without complication. Oxygen cannot be used alone as it is toxic under hyperbaric conditions (see below). Even remarkably inert gases (such as nitrogen) have toxic properties in hyperbaric conditions. There is no ideal gas mixture and the choice of diving gas is determined by balancing the physiological effects of each gas and the diving challenge (depth and duration). The commonly used gas mixtures include compressed air, oxygen, nitrox (nitrogen-oxygen mix), heliox (helium-oxygen) and trimix (helium, oxygen, nitrogen). The characteristics of these gases and their common uses are outlined in table 1.

Below $50 \mathrm{~m}$, helium-oxygen or helium-nitrogen-oxygen mixtures usually replace air as a breathing gas, both to avoid inert gas narcosis and to reduce breathing gas viscosity. Despite optimising the gas mixture, all diving gases have potentially dangerous toxic effects. An appreciation of these is essential for the diver to identify warning symptoms (if present). The clinical syndromes associated with dissolved gases are discussed below.

\section{Oxygen toxicity}

Oxygen is toxic under hyperbaric conditions. As a result, it is unsuitable as a pure breathing gas for all but very shallow dives and a carrier gas must be used with it. The risk of oxygen toxicity is dose dependent (depth, inspired oxygen fraction and duration) although there is considerable inter-individual and intra-individual variability in susceptibility. Long term hyperoxic exposure must generally be limited to around $0.4-0.5$ bar in order to avoid pulmonary oxygen toxicity, as applies in clinical practice. Short term oxygen exposure can be much higher but exposure to partial pressures greater than 1.6 atmospheres (atm) (>70 msw breathing air; $6 \mathrm{msw}$ breathing 100\% oxygen) may cause acute central nervous system (CNS) toxicity. The manifestations of this are legion and non-specific, but loss of consciousness is common and, in many cases, grand mal seizures occur without prodromal symptoms (box 1). ${ }^{6}$ Seizures spontaneously terminate upon cessation of oxygen inhalation but for unconscious divers who are not immediately rescued, drowning is almost inevitable.

Fortunately the threshold for CNS toxicity is higher for resting, dry exposures in the absence of hypercarbia and oxygen is regularly breathed at partial pressures up to 2.8 bar during the treatment of decompression illness and the provision of hyperbaric oxygen therapy.

\section{Nitrogen narcosis}

Nitrogen is narcotic when breathed under hyperbaric conditions. ${ }^{7}$ Nitrogen narcosis is characterised by euphoria, intoxication and progressive depression of central nervous system function (table 2). The onset is insidious and can result in irrational behaviour, impaired judgement and a false sense of security. Although there is considerable variation in individual susceptibility, performance is impaired in all individuals and short term adaptation to the narcotic effects does not occur. Many divers believe that they can develop resistance to nitrogen narcosis with practice but it has been shown that while habituation reduces subjective symptoms, performance remains impaired. ${ }^{8}$

Narcosis induced over confidence and impaired performance represents an important, and probably underestimated, threat to diver safety. In the Australasian diving fatality database,

Table 1 Properties of commonly used diving gases

\begin{tabular}{llll}
\hline Gas & Advantages & Disadvantages & Use \\
\hline Compressed air & Cheap and readily available & Nitrogen narcosis below $30 \mathrm{msw}$ & Most common breathing mixture for recreational \\
& & Density increases work of breathing below & diving \\
& & $50 \mathrm{msw}$ &
\end{tabular}

$100 \%$ oxygen

Minimal narcotic potency

CNS oxygen toxicity above 2 bar

Potential for oxygen toxicity if inappropriate mixture used at depth

(nitrogen-oxygen, Reduced decompression time

nitrogen $<80 \%$ )

Heliox

(helium-oxygen)

Reduced narcosis

Reduced density

High thermal conductivity

Speech distortion
High pressure neurological syndrome beneath $200 \mathrm{msw}$

Cost

Taste loss

$\begin{array}{ll}\text { Trimix } & \text { Reduced narcosis } \\ \text { (helium-nitrogen- } & \text { Reduced density } \\ \text { oxygen) } & \text { Reduced high pressure neurological syndrome } \\ & \text { risk on deep diving } \\ & \text { Avoid hyperoxia }\end{array}$

Cost

Complicated mixing and risk of error
Military and experimental divers Decompression gas in technical divers Limited to depths of 6-8 msw

Technical recreational diving Decompression gas for technical divers

Commercial diving $>50 \mathrm{msw}$ Military diving

Technical recreational diving

Deep commercial diving

Deep technical recreational diving 


\section{Box 1: Clinical features of oxygen toxicity}

\section{Acute oxygen toxicity symptoms}

- V: vertigo, visual disturbance

- E: ears, excitability, euphoria

- $\mathrm{N}$ : nausea, numbness

- T: tinnitus, twitching, tremor

- I: irritability, irrational behaviour

- D: dizziness, disorientation, depression

- C: convulsions

Project Stickybeak, it was estimated that nitrogen narcosis contributed to death in $9 \%$ of cases.

\section{High pressure neurological syndrome and helium}

Helium is used for deep diving because of an absence of narcotic effects. However, at depths below $120 \mathrm{~m}$ divers may develop "high pressure neurological syndrome" (HPNS), involving hyper-excitatory symptoms such as tremor, myoclonic jerks and irritability thought to be due to membrane and neurotransmitter mediated effects of pressure. ${ }^{910}$ The effects are reversible on return to normal ambient pressure but limit the performance of divers at extreme depths. Admixture of nitrogen into the heliox mixture, to create "trimix", provides some counter-acting narcotic effect that minimises this problem. ${ }^{11}$

\section{Contaminant gas toxicity}

The partial pressure of any breathing gas contaminant rises with increasing depth, mandating high standards of purity for breathing gas. Carbon monoxide poisoning has been responsible for many divers' deaths, and low molecular weight volatile hydrocarbon contamination is currently receiving attention as a possible cause of some cases of underwater incapacitation and post-dive malaise. ${ }^{12}$

Table 2 Typical clinical features of nitrogen narcosis

\begin{tabular}{ll}
\hline $\begin{array}{l}\text { Nitrogen partial } \\
\text { pressure (bar) }\end{array}$ & Symptoms and signs \\
\hline $2-4$ & $\begin{array}{l}\text { Mild impairment of performance of unpractised tasks } \\
\text { Mild euphoria }\end{array}$ \\
4 & $\begin{array}{l}\text { Impaired reasoning and immediate memory } \\
\text { Delayed response to visual and auditory stimuli } \\
\text { Increased reaction time }\end{array}$ \\
$4-6$ & $\begin{array}{l}\text { Overconfidence and fixed thinking } \\
\text { Calculation errors }\end{array}$ \\
6 & $\begin{array}{l}\text { Impaired judgement } \\
\text { Hallucinations }\end{array}$ \\
$6-8$ & $\begin{array}{l}\text { Laughter approaching hysteria } \\
\text { Talkative, occasional dizziness }\end{array}$ \\
8 & $\begin{array}{l}\text { Severely impaired intellectual performance } \\
\text { Mental confusion, impaired concentration }\end{array}$ \\
10 & Stupefaction \\
\hline 10 & Hallucinations, unconsciousness, death \\
\hline
\end{tabular}

\section{DIVING DISEASES: AETIOLOGY AND PATHOPHYSIOLOGY}

\section{Barotrauma}

Barotrauma can affect any non-vented gas containing space with damage occurring during compression ("squeeze") or due to gas expansion on ascent.

Descent of as little as $1-2 \mathrm{~m}$ is enough to cause pain, oedema and even haemorrhage (fig 2) in paranasal sinuses with blocked ostia or middle ears where there is Eustachian tube dysfunction. Middle ear barotrauma is the most common diving related pathology but is usually minor and self limiting. Tympanic membrane rupture can occur, however, and inner ear barotraumas with rupture of the round or oval window and perilymph fistula are particularly serious. Differential ear equalisation can result in the self explanatory and usually transient syndrome of alternobaric vertigo.

The records set by apnoea divers are remarkable with respect to pulmonary barotrauma, as a 20 -fold compression of the lung volume would probably result in fatal intrapulmonary haemorrhage for most untrained persons. Apnoea divers train to minimise their residual volume and also maximise their pre-dive total lung capacity by using an oropharyngeal "breath stacking" technique that overinflates the lung. Pursed lips expiration after surfacing creates positive expiratory pressure aimed at reducing the risk of pulmonary oedema that sometimes occurs. Some divers may develop cystic emphysematous lung changes as a result of hyperinflation over a prolonged period. ${ }^{13}{ }^{14}$

\section{Pulmonary barotrauma of ascent and arterial gas embolism}

Unlike breath-hold divers, divers using breathing apparatus maintain relatively normal pulmonary volumes while diving but during ascent, the compressed gas in their lungs expands with the falling ambient pressure. If intrapulmonary gas is prevented from escaping as a result of a closed glottis, bronchospasm or gas trapping, excessive transpulmonary pressures will result. Differential alveolar compliance results in differential expansion of adjacent lung units causing focal shearing between vessels and airways and rupture of small airways and/or alveoli. Escaping gas can enter the pleural cavity (pneumothorax), mediastinum (mediastinal emphysema that may extend down into the retroperitoneum or appear around the neck as subcutaneous emphysema), pericardial cavity (pneumopericardium) or pulmonary venules (which transits the left heart to

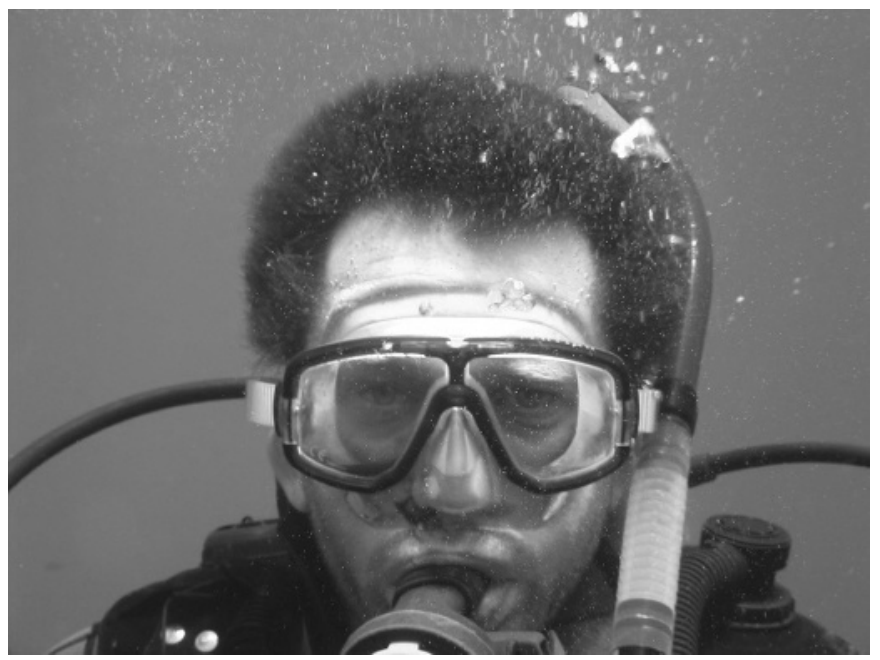

Figure 2 A diver with sinus barotraumas-blood is seen in the mask from sinus haemorrhage. 
result in arterial gas embolism). The subsequent pathophysiological processes and clinical presentation are similar to those caused by iatrogenic gas embolism such as may occur during laparoscopic surgery or cardiopulmonary bypass.

Gas bubbles entering the systemic circulation are distributed by buoyancy and blood flow. The cerebral circulation and the middle cerebral artery in particular are common sites of bubble distribution. Most bubbles pass through the cerebral vasculature after varying delay, although sustained occlusion resulting in infarction may occur. ${ }^{15}$ Intravascular bubbles trigger an acute inflammatory response with haematological and vascular components, which may result in reduced cerebral blood flow, decreased integrity of the blood-brain barrier and cerebral oedema.

Cerebral arterial gas embolism (CAGE) classically presents within seconds to minutes of a diver surfacing, with profound neurological symptoms which may resemble a cerebrovascular accident. Clinical features include paralysis, convulsions and coma, often accompanied by cardiovascular instability or cardiac arrest. Most cases improve partially or completely over a short period as blood flow is re-established, but relapse is common over the hours following due to re-embolisation or post-embolism vascular leak and inflammation.

Box 2 lists the various types of barotraumas.

\section{Decompression sickness: "The bends"}

During a dive, inert gas becomes dissolved in tissues as a function of time and depth. After many hours, a state of equilibrium can be reached between the breathing gas and the tissues, known as saturation. As divers ascend to the surface, the nitrogen or helium diffuses from tissues into the blood and from blood into the lungs. As the partial pressure of inert gas in the blood and tissues exceeds ambient pressure, bubbles form in the tissues and blood vessels, which may result in the clinical syndrome of decompression sickness.

The clinical manifestations of decompression sickness are protean, reflecting the effects of bubble formation in diverse anatomical locations (table 3). Multiple systems may be involved. Symptoms may occur immediately on surfacing, or onset may be delayed for up to $48 \mathrm{~h}$. The disease may be progressive or may stabilise or can be relapsing and remitting in nature. It may be minor and self limiting in nature, but can be

Box 2: Types of barotrauma

\section{Ear, nose and throat}

- Mask squeeze

- Sinus barotrauma

- Tooth barotrauma

- Middle ear barotrauma

- Inner ear barotrauma

Gastrointestinal tract

- Hollow viscus perforation

Pulmonary barotrauma

- Surgical emphysema

- Pneumomediastinum

- Pneumothorax \pm tension

Arterial gas embolism

- Coronary artery gas embolism

- Cerebral artery gas embolism catastrophic resulting in permanent paralysis and death. Altitude has a significant triggering and exacerbating effect and emergency transport of divers should avoid significant altitude exposure.

Bubbles exert their pathophysiological effects by mechanically distorting tissues, obstructing blood flow and initiating an inflammatory response.

Doppler ultrasound has demonstrated that decompression sickness is associated with venous intravascular bubbles and that the bubble load is proportional to the decompression stress. ${ }^{16}{ }^{17}$ However, it should be emphasised that the presence of bubbles is not always associated with overt clinical symptoms and many, if not most, dives result in "silent" bubbles.

The majority of intravascular bubbles in decompression sickness occur in the venous circulation. The lungs act as a filter, trapping and excreting venous bubbles and preventing their passage into the systemic circulation. Exceptions to this rule occur in severe decompression sickness where the pulmonary circulation is overwhelmed by the bubble load "the chokes"), in right-to-left intracardiac shunts (for example, patent foramen ovale (PFO)), via intrapulmonary shunting and as a result of pulmonary barotrauma, where bubbles directly enter the pulmonary circulation. In these cases bubbles enter the arterial circulation resulting in gas embolism with the consequences discussed previously. Of particular recent interest has been the observation that individuals with large PFOs have an increased risk of neurological decompression sickness and particularly spinal cord disease..$^{18}$

Intravascular bubbles damage both the blood vessel luminal surfactant layer and endothelial cells. ${ }^{19}{ }^{20}$ This reduces the integrity of the vessels and causes endothelial activation. Bubbles also interact with formed elements of blood and plasma proteins. They may cause platelet and leucocyte aggregation, cytokine release, and activate the complement, kinin, fibrinolytic and coagulation cascades. The subsequent acute inflammatory response results in increased capillary permeability and haemoconcentration. ${ }^{21}$

\section{Pulmonary oedema}

Acute pulmonary oedema is a rare but dramatic result of some or all of a group of factors that can increase pulmonary vascular distension and generate negative airways pressures. Immersion, cold, vigorous exercise, hyperventilation, poorly functioning breathing regulators and fluid overload as a result of over zealous attempts to avoid dehydration have all been implicated. ${ }^{22}$ There may be a common constitutional risk factor with hypertension as non-hypertensives who experience an episode may be predisposed to developing hypertension in the years that

Table 3 Clinical features and incidence in decompression sickness: data from 1170 cases recorded in the Institute of Naval Medicine, UK between 1990 and 1999 (personal communication)

\begin{tabular}{ll}
\hline Manifestation & Prevalence (\%) \\
\hline Neurological symptoms (including & 77 \\
auditory) & \\
Limb pain & 48 \\
Constitutional & 29 \\
Skin & 9.7 \\
Respiratory & 3.7 \\
Girdle/back pain & 3.2 \\
Lymphatic & 0.9 \\
\hline
\end{tabular}


follow. ${ }^{23}$ Differential changes in right versus left sided cardiac contractility have also been hypothesised as contributing. The syndrome usually resolves rapidly but may be the cause of some deaths attributed to drowning

\section{Long term effects of diving}

Diving appears generally well tolerated, but regular divers probably self select as "healthy survivors". In contrast, susceptible individuals or those who expose themselves to less controlled diving can experience neurocognitive dysfunction, dysbaric osteonecrosis, hearing loss and pulmonary function changes. ${ }^{24}$ The Evaluation of Long Term Health Impacts of Diving (ELTHI diving study) project undertaken by Aberdeen University on behalf of the UK Health and Safety Executive has compared the long term health of offshore oil industry divers with other offshore workers and found some increase in self reported complaints of forgetfulness, loss of concentration, hearing loss and musculoskeletal symptoms among divers; however, overall health quality of life was not significantly different from other workers and the overall findings did not exceed the threshold of "clinical significance". ${ }^{25}$ Subsequent analysis has suggested some association between the reported deficits and underwater welding work but it remains a possibility that at least some individuals can accrue symptomatic health decrements as a result of diving, even when overt decompression illness has not occurred. ${ }^{26}$

\section{PREVENTION AND TREATMENT OF DIVING DISEASE Avoiding decompression sickness}

Divers use various forms of decompression procedures to minimise the risk of decompression sickness. Dive computers, published tables and personal computer software algorithms recommend limits on dive duration for any given depth and prescribe maximum ascent rates and decompression stops for various combinations of depth, dive time and breathing gas mixture. Repetitive dives and dives at altitude require more conservative exposures. Decompression models are variously based on theoretical exponential wash-in and washout curves for inert gas uptake and excretion by the tissues and/or data from both research and field dives. Many models involve very complex and relatively poorly validated mathematics, but despite this, implementation of the most common approaches has effected both recreational and occupational decompression with illness rates as low as 1:5000 dives or less where diving is undertaken in well controlled and non-experimental settings. ${ }^{1}$

There seems to be pronounced inter-individual variability in susceptibility to decompression sickness as well as many factors that can vary the risk for any one individual. As a consequence, some individuals will experience symptoms even if they strictly follow established decompression procedures. Factors associated with an increased risk of decompression sickness include obesity, fatigue, cold, dehydration, and inter-current illness. ${ }^{27}$ Regular diving may produce tolerance in some individuals while preconditioning via vigorous exercise shows some promise, although this may only be protective within specific pre-dive time windows. ${ }^{28}$

\section{Treatment for decompression sickness and gas embolism}

In addition to normal first aid procedures, oxygen administration is a priority. Oxygen not only treats arterial hypoxaemia but also enhances the rate of elimination of inert gas and the resolution of bubbles. During transport and treatment, divers should be kept in a supine position to minimise the risk of further cerebral artery embolism. Intravenous fluid resuscitation is recommended to counteract the intravascular depletion and compromised microcirculation caused by pressure induced diuresis, endothelial damage and increased capillary permeability. In the absence of intravenous fluids, oral rehydration is recommended.

The definitive treatment is recompression in a hyperbaric chamber. Early treatment results in a better prognosis. ${ }^{1}$ Compression physically reduces bubble volume in accordance with Boyle's law. The use of $100 \%$ oxygen as the breathing gas during recompression is therapeutic via various mechanisms including rapid elimination of inert gas, maximal oxygenation of ischaemic tissues, reduction of oedema, and inhibition of secondary inflammatory and reperfusion injury, including via inhibition of neutrophil adhesion molecule expression. ${ }^{29}$

Recompression schedules for the treatment of decompression sickness consist of a rapid recompression to a specified pressure with oxygen breathing interrupted periodically by "air breaks" and continuing during a subsequent slow and staged decompression. The most commonly used initial schedule ("USN6" or "RN62") takes approximately $5 \mathrm{~h}$. If symptoms do not resolve completely, treatments are repeated once or twice daily, until the symptoms are relieved or a plateau in improvement is reached. The vast majority of patients only require one to three treatments. ${ }^{30}$

A number of adjunctive treatments have been used in decompression sickness. A recent double blind randomised controlled trial indicates that non-steroidal anti-inflammatory drugs can reduce the number of recompression sessions required, although symptomatic outcome was not improved. ${ }^{31}$ Steroids and aspirin are sometimes used but appear not to improve outcome. ${ }^{32}$ Similarly anticoagulation with heparin is probably not beneficial and may increase the risk of spinal cord haemorrhage. ${ }^{33}$

An interesting development has been the use of lignocaine in acute decompression sickness. Lignocaine has been shown to reduce brain dysfunction after air embolism in animal models. ${ }^{34}$ Several case reports suggest that lignocaine has a beneficial effect on outcome in decompression sickness, even if treatment has been delayed and the symptoms had been previously refractory to recompression. ${ }^{35}{ }^{36}$ Putative mechanisms for the efficacy of lignocaine include an anti-leucocyte effect and the deceleration of ischaemic transmembrane ion shifts. ${ }^{37} \mathrm{~A}$ controlled trial is awaited.

Box 3 lists the decompression sickness hotlines in the UK.

\section{NEW DEVELOPMENTS AND POTENTIAL TRANSLATIONAL RESEARCH RELEVANT TO MEDICINE}

In recent years, sophisticated electronically controlled mixed gas rebreather systems have become available for advanced recreational divers, enabling untethered diving to depths well in excess of the normal recreational range. This has brought about a range of new computer algorithms for calculating decompression based upon widely varying datasets, underlying mathematics and physiological assumptions. Some incorporate much deeper decompression stops than used traditionally, but it is far from clear whether this is advantageous. An analysis of this practice will be reported in the proceedings of an expert workshop on the subject that was run by the Divers Alert Network in June 2008.

With regard to medical fitness to dive, traditional deterministic views on who is fit to dive are being challenged by permissive risk assumption philosophies and this, combined with an accumulation of data on uneventful dives by divers with health problems, has led to many contraindicationspreviously held to be absolute-becoming relative. In particular, 
Box 3: Decompression sickness hotlines in the UK and a selection of international diving medical societies

- Scottish Diving Hotline, Aberdeen Royal Infirmary: 0845408 6008 (24 h)

- English Diving Hotline, Institute of Naval Medicine: +44 7831 151523 (24 h)

- British Hyperbaric Association: www.hyperbaric.org.uk (UK chamber information)

- South Pacific Underwater Medicine Society: www.spums.org.au

- Undersea and Hyperbaric Medicine Society: www.uhms.org

- European Underwater and Baromedical Society: www.eubs.org

- DAN: Divers Alert Network: www.diversalertnetwork.org

- UK Sport Diving Medical Committee: www.uksdmc.co.uk

some diabetic and asthmatic subjects are now undertaking scuba diving, principally in the recreational setting. ${ }^{38}$ It is hoped that more sophisticated registries will be developed to provide a better evidence base for estimating the significance of such risks.

The possibility of there being gender differences in decompression illness risk has long been a subject of speculation. A prospective trial of altitude exposure showed no net differences between men and women in risk for altitude decompression illness, but there was some association with menstrual cycle phase in subjects taking oral contraceptives. ${ }^{39}$ Prospective data on over 34000 recreational dives by women submitting dive data to an ongoing project by the Diving Diseases Research Centre also suggests an association of risk with phase of the menstrual cycle, with problems more likely to be reported during the last and first weeks of the cycle. ${ }^{40}{ }^{41}$ With the trauma literature suggesting that female sex hormones play a modulating role in secondary inflammatory processes after trauma, there is clear potential for further work in this field. ${ }^{42}{ }^{43}$

Preconditioning, oxidant stress and secondary injury modulation are active research areas that may produce results relevant to divers. Likewise, divers may prove a testing ground for therapeutic substances. Decompression illness treatments, which target the secondary effects of bubbles, are currently a focus of research. Modification of leucocyte behaviour in particular may become a major therapeutic modality in the future. Monoclonal antibodies to leucocyte adhesion molecules are currently being investigated in animal models of decompression illness. ${ }^{44}$ Perfluorocarbons have the potential to enhance off-gassing while providing favourable oxygen delivery and possibly useful surfactant and immunomodulatory effects. ${ }^{45}$

A preconditioning agent of particular interest is oxygen breathed under pressure. Rapidly evolving research with hyperbaric oxygen demonstrates that the oxidative stress this therapy provides can upregulate endogenous antioxidants, moderate inflammatory injury and inhibit reperfusion injury and apoptosis in a variety of settings. ${ }^{46-51}$ Exposure to hyperbaric oxygen exposure before diving shows potential to reduce the risk of decompression illness. ${ }^{52-54}$ If they were able to be accessed, the growing pool of nitrox and rebreather divers could provide data on a natural human experiment that is testing both tolerance to prolonged hyperoxia and the interaction of hyperoxia with decompression illness risk.

The apparently dramatic inter-individual variations in susceptibility to diving illnesses and oxygen toxicity are almost certainly the result of constitutional and genetically determined factors. As exposure to the physiological stress of diving is common, divers could provide a fruitful source of material for genomic and proteomic analysis against markers such as Doppler bubble scoring and cytokines or other markers of endothelial injury.

In the diving oriented basic science laboratory, much useful oxygen toxicity research continues; one particularly interesting recent finding is that pulmonary oxygen toxicity at pressure appears modulated via neurological pathways that are quite different to the oxygen toxicity generated by prolonged ventilation on high fractional inspired oxygen $\left(\mathrm{FiO}_{2}\right)$ values at sea level. ${ }^{55}$ With respect to the molecular mechanisms of inert gas narcosis and HPNS, much remains unknown but there is almost certainly common ground with mechanisms of anaesthesia and the toxicity of various gases. Investigations into the roles of micronuclei, caveoli, surfactants and the nature and associations of endothelial microparticles seem likely to enhance our understanding of not only decompression illness but also how to minimise the risks of cognitive impairment and other side effects of cardiac bypass and procedures associated with the potential for gas embolism.

The hyperbaric environment presents an opportunity to study normal physiological systems under extreme environmental stress, giving an insight into the limits of physiological tolerance. The pathophysiology of diving related syndromes such as decompression illness, nitrogen narcosis and oxygen toxicity may provide insight into the pathological processes underlying clinical situations such as acute lung injury, systemic inflammatory response syndrome, mechanisms of anaesthesia and post-anaesthetic and post-cardiac bypass morbidity.

\section{SUMMARY}

The underwater environment presents a number of challenges, both physical and physiological, to the diver. Diving is associated with multiple risks, some potentially fatal including drowning, hypothermia, inert gas narcosis, oxygen toxicity, arterial gas embolism, decompression sickness, high pressure neurological syndrome, chronic joint dysfunction and neurocognitive impairment. In spite of these risks, it is an increasingly popular pastime and the boundaries of both recreational and occupational diving are continuously being expanded. Consequently, general physicians may encounter diving related pathology in their acute medical practice and an understanding of diving pathophysiology is increasingly important. As more patients with medical comorbidity undertake diving, an appreciation of the implications of chronic medical conditions for diving will also become valuable. Finally, research in extreme environments allows exploration of human physiology at its limits. This has the potential to provide valuable insights into pathophysiology and medicine and has potential translational relevance to the clinical arena.

\section{Key learning points}

- There is wide inter-individual variability in susceptibility to decompression illness.

- Supplemental oxygen and rapid transfer to a recompression facility are the treatment priorities in diving related illness.

- Prevention of decompression illness by safe diving practice is key.

- Inflammation is an important mechanism of injury in bubble related disease.

- Gas embolism may benefit from hyperbaric oxygen treatment.

- Breathing oxygen under conditions of increased pressure is potentially toxic. 


\section{Key references}

- Brubakk A, Neumann T, eds. Bennett and Elliot's physiology and medicine of diving. London: Elsevier Science, 2003.

- Macdiarmid JI, Ross JAS, Taylor CL, et al. Coordinated investigation into the long term health effects of diving (the ELTHI Diving Study). UK Health and Safety Executive Report Research Report 230 2004. www.hse.gov.uk/research/rrpdf/ rr230.pdf

- Bennett M, Mitchell S, Dominguez A. Adjunctive treatment of decompression illness with a non-steroidal anti-inflammatory drug (tenoxicam. Undersea Hyperb Med 2003;30:195-205.

- Mitchell SJ. Lidocaine in the treatment of decompression illness: a review of the literature. Undersea Hyperb Med 2001;28:165-74.

- Dujic Z, Valic Z, Brubakk A0. Beneficial role of exercise on scuba diving. Exercise and Sport Sciences Reviews 2008;36:38-42.

\section{Competing interests: None declared.}

\section{REFERENCES}

1. Vann RD, Freiberger JJ, Caruso JL, et al. Report on decompression illness, diving fatalities and project diver exploration. DAN's Annual Review of Recreational Scuba Diving 2005;1.

2. International Association for the Development of Free Diving www.aida-international.org

3. Gardette B, Delauze HG. [Techniques of underwater intervention: means, methods, research and outlook]. Bull Acad Natl Med 1996;180:975-83.

4. Benuet PB, Elliot DH, eds. The physiology and medicine of diving. Balliere Tindall, 1982:11.

5. Camporesi EM, Bosco G. In: Brubakk A, Neuman T, eds. Bennet and Elliott's physiology and medicine of diving. London: Elsevier Science, 2003:95-96.

6. Donald KW. Oxygen poisoning in man I and II. BMJ 1947;i:667-72.

7. Motley A. The psychologic effects from breathing air at 4 atmospheres pressure. Am J Physiol 1935;112:554-8.

8. Hamilton K, Laliberte MF, Fowler B. Dissociation of the behavioral and subjective components of nitrogen narcosis and diver adaptation. Undersea Hyperb Med 1995;22:41-9.

9. Jain KK. High-pressure neurological syndrome (HPNS). Acta Neurol Scand 1994; $90: 45-50$.

10. Talpalar AE, Grossman Y. CNS manifestations of HPNS: revisited. Undersea Hyperb Med 2006;33:205-10

11. Bennett PB, Rostain JC. In: Brubakk A, Neuman T, eds. Bennet and Elliott's physiology and medicine of diving. London: Elsevier Science, 2003:323-57.

12. Millar IL, Mouldey PG. Compressed breathing air - the potential for evil from within. Diving and Hyperbaric Medicine 2008;38:145-8.

13. Ferretti G. Extreme human breath-hold diving. Eur J Appl Physiol 2001;84:254-71.

14. Pollock NW. Breath-hold diving - performance and safety. Diving and Hyperbaric Medicine 2008;38:79-86.

15. Gorman DF, Browning DM. Cerebral vasoreactivity and arterial gas embolism. Undersea Biomed Res 1986;13:317-35.

16. Reinertsen RE, Flook V, Koteng $S$, et al. Effect of oxygen tension and rate of pressure reduction during decompression on central gas bubbles. J App/ Physiol 1998;84:351-6.

17. Spencer MP, Campbell SD, Sealey JL, et al. Experiments on decompression bubbles in the circulation using ultrasonic and electromagnetic flowmeters. J Occup Med 1969;11:238-44.

18. Wilmshurst $\mathbf{P}$, Bryson P. Relationship between the clinical features of neurological decompression illness and its causes. Clin Sci (Lond) 2000;99:65-75.

19. Haller C, Sercombe R, Verrecchia C, et al. Effect of the muscarinic agonist carbachol on pial arteries in vivo after endothelial damage by air embolism. J Cereb Blood Flow Metab 1987;7:605-11.

20. Hills BA, James PB. Microbubble damage to the blood-brain barrier: relevance to decompression sickness. Undersea Biomed Res 1991;18:111-16.

21. Boussuges A, Blanc P, Molenat F, et al. Haemoconcentration in neurological decompression illness. Int J Sports Med 1996;17:351-5.

22. Koehle MS, Lepawsky M, McKenzie DC. Pulmonary oedema of immersion. Sports Med 2005;35:183-90.

23. Wilmshurst PT, Nuri M, Crowther A, et al. Cold-induced pulmonary oedema in scuba divers and swimmers and subsequent development of hypertension. Lancet 1989;i:62-5.
24. Dutka AJ. In: Brubakk A, Neuman T, eds. Bennet and Elliott's physiology and medicine of diving. London: Elsevier Science, 2003:680-99.

25. Macdiarmid JI, Ross JAS, Taylor CL, et al. Coordinated investigation into the long term health effects of diving (the ELTHI Diving Study). UK Health and Safety Executive Report Research Report 230 2004. www.hse.gov.uk/research/rrpdf/rr230.pdf

26. Macdiarmid JI, Ross JAS, Semple S, et al. Further investigation of possible musculoskeletal and cognitive deficit due to welding in divers identified in the ELTHI Diving Study. UK Health and Safety Executive Report 390, 2005. www.hse.gov.uk/ research/rrpdf/rr390.pdf

27. Brubakk A, Neuman T, eds. Bennet and Elliott's physiology and medicine of diving Saunders, 2003.

28. Dujic Z, Valic Z, Brubakk A0. Beneficial role of exercise on scuba diving. Exercise and Sport Sciences Reviews 2008;36:38-42.

29. Helps SC, Gorman DF. Air embolism of the brain in rabbits pretreated with mechlorethamine. Stroke 1991;22:351-4.

30. Dankner R, Gall N, Freidman G, et al. Recompression treatment of Red Sea diving accidents: a 23-year summary. Clin J Sport Med 2005;15:253-6.

31. Bennett M, Mitchell S, Dominguez A. Adjunctive treatment of decompression illness with a non-steroidal anti-inflammatory drug (tenoxicam. Undersea Hyperb Med 2003;30:195-205

32. Bove AA. The basis for drug therapy in decompression sickness. Undersea Biomed Res 1982; 9:91-111.

33. Palmer AC, Blakemore WF, Payne JE, et al. Decompression sickness in the goat: nature of brain and spinal cord lesions at 48 hours. Undersea Biomed Res 1978; 5:275-86

34. Evans DE, Kobrine Al, LeGrys DC, et al. Protective effect of lidocaine in acute cerebral ischemia induced by air embolism. J Neurosurg 1984;60:257-63.

35. Cogar WB. Intravenous lidocaine as adjunctive therapy in the treatment of decompression illness. Ann Emerg Med 1997;29:284-6.

36. Drewry A, Gorman DF. Lidocaine as an adjunct to hyperbaric therapy in decompression illness: a case report. Undersea Biomed Res 1992;19:187-90.

37. Mitchell SJ. Lidocaine in the treatment of decompression illness: a review of the literature. Undersea Hyperb Med 2001;28:165-74.

38. Harrison D, Lloyd-Smith R, Khazei A, et al. Controversies in the medical clearance of recreational scuba divers: updates on asthma, diabetes mellitus, coronary artery disease, and patent foramen ovale. Curr Sports Med Rep 2005:4:275-81.

39. Webb JT, Kannan N, Pilmanis AA. Gender not a factor for altitude decompression sickness risk. Aviat Space Environ Med 2003;74:2-10.

40. Lee V, St Leger Dowse M, Edge C, et al. Decompression sickness in women: a possible relationship with the menstrual cycle. Aviat Space Environ Med 2003;74:1177-82.

41. St Leger Dowse M, Gunby A, Phil D, et al. Problems associated with scuba diving are not evenly distributed across a menstrual cycle. J Obstet Gynaecol 2006;26:216-21.

42. Choudhry MA, Bland $\mathrm{Kl}$, Chaudry $\mathrm{IH}$. Trauma and immune response - effect of gender differences. Injury 2007;38:1382-91.

43. Hsieh YC, Frink M, Choudhry MA, et al. Metabolic modulators following trauma sepsis: sex hormones. Crit Care Med 2007;35:S621-9.

44. Nossum V, Hjelde A, Bergh K, et al. Anti-C5a monoclonal antibodies and pulmonary polymorphonuclear leukocyte infiltration - endothelial dysfunction by venous gas embolism. Eur J Appl Physiol 2003;89:243-8.

45. Shaffer TH, Forman DL, Wolfson MR. Physiological effects of ventilation with liquid fluorocarbon at controlled temperatures. Undersea Biomed Res 1984;11:287-98.

46. Li J, Liu W, Ding S, et al. Hyperbaric oxygen preconditioning induces tolerance against brain ischemia-reperfusion injury by upregulation of antioxidant enzymes in rats. Brain Res 2008;1210:223-9.

47. Li 0, Li J, Zhang L, et al. Preconditioning with hyperbaric oxygen induces tolerance against oxidative injury via increased expression of heme oxygenase-1 in primary cultured spinal cord neurons. Life Sci 2007:80:1087-93.

48. Li Z, Liu W, Kang Z, et al. Mechanism of hyperbaric oxygen preconditioning in neonatal hypoxia-ischemia rat model. Brain Res 2008;1196:151-6.

49. Ostrowski RP, Graupner G, Titova E, et al. The hyperbaric oxygen preconditioning induced brain protection is mediated by a reduction of early apoptosis after transient global cerebral ischemia. Neurobiol Dis 2008;29:1-13.

50. Peng Z, Ren P, Kang Z, et al. Up-regulated HIF-1alpha is involved in the hypoxic tolerance induced by hyperbaric oxygen preconditioning. Brain Res 2008;1212:71-8.

51. Yogaratnam JZ, Laden G, Guvendik L, et al. Pharmacological preconditioning with hyperbaric oxygen: can this therapy attenuate myocardial ischemic reperfusion injury and induce myocardial protection via nitric oxide? J Surg Res 2008:149:155-64.

52. Arieli Y, Katsenelson K, Arieli R. Bubble reduction after decompression in the prawn Palaemon elegans by pretreatment with hyperbaric oxygen. Undersea Hyperb Med 2007:34:369-78.

53. Butler BD, Little T, Cogan V, et al. Hyperbaric oxygen pre-breathe modifies the outcome of decompression sickness. Undersea Hyperb Med 2006;33:407-17.

54. Katsenelson K, Arieli Y, Abramovich A, et al. Hyperbaric oxygen pretreatment reduces the incidence of decompression sickness in rats. Eur J Appl Physiol 2007; 101:571-6.

55. Demchenko IT, Welty-Wolf KE, Allen BW, et al. Similar but not the same: normobaric and hyperbaric pulmonary oxygen toxicity, the role of nitric oxide. Am J Physiol Lung Cell Mol Physiol 2007;293:L229-38. 